\title{
Article \\ PROX1, a Key Mediator of the Anti-Proliferative Effect of Rapamycin on Hepatocellular Carcinoma Cells
}

\author{
Sora Kwon ${ }^{1}$, Kiwon Ban ${ }^{2}$, , Young-Kwon Hong ${ }^{3}$, Jung-Suk Sung ${ }^{4}$ and Inho Choi ${ }^{1, *}$ \\ 1 Department of Pharmaceutical Engineering, Hoseo University, Asan 31499, Korea; kwon_sora_@naver.com \\ 2 Department of Biomedical Sciences, City University of Hong Kong, Hong Kong 999077, China; \\ Ban.KW@cityu.edu.hk \\ 3 Department of Surgery, Norris Comprehensive Cancer Center, Keck School of Medicine, \\ University of Soutern California, Los Angeles, CA 90033, USA; young.hong@usc.edu \\ 4 Department of Life Science, Dongguk University, Goyang 10326, Korea; sungjs@dongguk.edu \\ * Correspondence: inhochoi@hoseo.edu
}

Citation: Kwon, S.; Ban, K.; Hong, Y.-K.; Sung, J.-S.; Choi, I. PROX1, a Key Mediator of the

Anti-Proliferative Effect of

Rapamycin on Hepatocellular

Carcinoma Cells. Cells 2022, 11, 446

https://doi.org/10.3390/

cells11030446

Academic Editor: Ali Canbay

Received: 21 December 2021

Accepted: 25 January 2022

Published: 27 January 2022

Publisher's Note: MDPI stays neutral with regard to jurisdictional claims in published maps and institutional affiliations.

Copyright: (c) 2022 by the authors. Licensee MDPI, Basel, Switzerland. This article is an open access article distributed under the terms and conditions of the Creative Commons Attribution (CC BY) license (https:// creativecommons.org/licenses/by/ $4.0 /)$.

\begin{abstract}
The MTOR signal is known to be activated in various cancer cells including hepatocellular carcinoma (HCC) cells. Rapamycin, a specific inhibitor of MTOR, has been widely used as an immunosuppressant in organ transplant patients, and its clinical application has been recently expanded to cancer therapy. In this study, the anti-proliferative effect of rapamycin was investigated in four different HCC cell lines. Rapamycin effectively inhibited the proliferation of Huh7 or Hep3B, but not that of HepG2 or SNU3160 cells. Interestingly, rapamycin increased Prospero-related homeobox 1 (PROX1) expression at the protein level, but did not affect its transcript in Huh7 as well as Hep3B cells. Moreover, immunoprecipitation assays showed that PROX1 ubiquitination was downregulated by rapamycin. Furthermore, PROX1 over-expression or siRNA knock-down in Huh7 and Hep3B cells reduced or increased proliferation, respectively. The effect of PROX1 over-expression on the sensitivity to rapamycin was not synergistic, but the effect of MTOR inhibition on cell proliferation was diminished by PROX1 siRNA. Finally, Huh7 cells were inoculated into the flanks of nude mice and rapamycin was injected daily for 14 days. The xenograft volume was decreased and PROX1 expression was increased by rapamycin. These results indicate that PROX1 plays a key role in the anti-proliferative effect of rapamycin and suggest that the increased PROX1 by MTOR inhibition can be used as a useful marker for predicting whether HCC cells can be affected by rapamycin.
\end{abstract}

Keywords: hepatocellular carcinoma; MTOR; rapamycin; PROX1; antiproliferation

\section{Introduction}

Hepatocellular carcinoma (HCC) is one of the most common malignant cancers [1] and the third leading cause of cancer-related deaths worldwide [2]. However, only $30 \%$ of HCC patients can be treated by liver resection, transplantation, or local ablation, mostly owing to its heterogeneous etiology. Regarding chemotherapy, a small number of drugs are available to treat HCC patients [3]. Unfortunately, recent clinical trials have shown that sorafenib is not very effective for all HCC patients. The results from the SHARP (Sorafenib HCC Assessment Randomized Protocol) trial demonstrated that the overall survival (OS) prolongation of HCC patients receiving sorafenib was only 2.8 months, and that objective tumor response occurred in only a small proportion of patients $(0.6 \%$ to $2 \%$ ) [4]. Recently, lenvatinib, a multikinase inhibitor, was approved for the treatment of advanced or unresectable HCC patients who had not received prior systemic therapy. However, OS proportions of patients treated with Lenvatinib were only $18 \%$. Thus, it is highly required to identify molecular markers that predict the patients likely to benefit from sorafenib or lenvatinib chemotherapy [5,6].

Recently, it was reported that the mammalian target of rapamycin (MTOR, a serinethreonine kinase of the phosphatidylinositol (PI) kinase-related protein kinase family [7]) is 
activated in various carcinoma cells, including breast cancer [8], ovarian cancer [9], and HCC [10]. MTOR regulates cell growth and proliferation by controlling translation of several proteins, such as cyclin dependent kinase inhibitor p27kip1, retinoblastoma protein, cyclin D1, c-myc, or STAT3 [11]. The MTOR signal can be activated by various stimuli such as growth factors, nutrients through receptor tyrosine kinase (RTK), phosphatidyl inositol 3 kinase (PI3K), and the Akt/PKB signaling cascade [12]. The MTOR signal elicits its effect by binding to the cytosolic immunophilin FKBP12 (FK506 binding protein, $12 \mathrm{kDa}$ ) [13]. Owing to its activation in cancer cells, specific inhibitors of MTOR including rapamycin have been considered as attractive reagents for cancer therapy [11]. Rapamycin, also known as sirolimus, shows its unique inhibitory effects by binding to MTOR and has been used extensively in transplantation to prevent organ rejection $[14,15]$, and recently, its application has expanded to cancer therapy [16].

It was recently reported that Prospero-related homeobox 1 (PROX1), an important transcription factor responsible for the development of several tissues such as lymphatics or liver [17-19], contributes to cancer development and progression. PROX1 has been ascribed tumor suppressive and oncogenic properties in different cancer types [20]. Liu et al. [21] reported that PROX1 promotes HCC cell proliferation, but it was also demonstrated that PROX1 can inhibit the proliferation of hepatocellular or oral carcinoma cells [22,23].

Despite the significant roles of MTOR and PROX1 in the proliferation of carcinoma cells, few studies have investigated the relationship between these two factors. Thus, we examined the anti-proliferative effect of rapamycin on HCC and its relation to PROX1 expression in four different HCC cell lines: Huh7, Hep3B, HepG2, and SNU3160. Interestingly, we found that rapamycin reduced the proliferation of Huh7 or Hep3B cells, but not that of HepG2 or SNU3160 cells. Moreover, rapamycin significantly increased PROX1 expression in Huh7 and Hep3B cells by increasing the half-life of PROX1 protein by reducing its ubiquitination without affecting PROX1 transcript.

Subsequent functional studies using PROX1 knock-down or over-expression showed that PROX1 is a critical mediator of the anti-cancer effect of rapamycin. Furthermore, in an in vivo study using Huh7 xenograft, rapamycin reduced xenograft volume and increased PROX1 expression in tumors. Together, these results indicate that PROX1 plays an important role in the anti-proliferative effect of rapamycin and suggest that PROX1 protein level might be a useful marker for anti-cancer therapy of rapamycin in HCC.

\section{Materials and Methods}

\subsection{HCC Cell Lines and Reagents}

Human HCC cell lines, Huh7, Hep3B, HepG2, and SNU3160 (purchased from the Korean Cell Line Bank, Seoul National University, Seoul, Korea), were cultured in Dulbecco's modified Eagle medium (DMEM) with 10\% fetal bovine serum (FBS), 5\% penicillinstreptomycin, and $5 \%$ sodium pyruvate, in an atmosphere containing $5 \% \mathrm{CO}_{2}$ at $37^{\circ} \mathrm{C}$. Cells were treated with or without rapamycin ( $10 \mathrm{nM}$, LC Laboratories, Woburn, MA, USA) in low serum media (1\% FBS) for $48 \mathrm{~h}$, unless otherwise indicated. Cells were transfected with the small interfering RNA (siRNA) against PROX1 (forward: 5'-GCAAAGAUGUUGAUCCUU CTT-3' and reverse: 5'-GAAGGAUCAACAU-CUUUGCTT-3') for knock-down experiment or the over-expression system of PROX1 (human PROX1 coding sequence in pcDNA3, kindly provided by Prof. Y-K Hong, University of Southern California) using Lipofactor2000 (Aptabio, Korea). To measure PROX1 protein stability, cells were treated with cycloheximide (CHX, Sigma-Aldrich, San Diego, CA, USA) for up to $6 \mathrm{~h}$ after incubation for $24 \mathrm{~h}$ with or without rapamycin.

\subsection{Cell Proliferation Assay}

Cell proliferation was analyzed using the Premixed WST-1 Cell Proliferation Assay kit (TaKaRa, Tokyo, Japan), as described previously [24]. In brief, cells (Huh7, $2 \times 10^{4}$ cells /well; HepG2, Hep3B, and SNU3160 cells, $3 \times 10^{4}$ cells / well) were seeded in 24-well plates (SPL Inc., Pyeongtaek, Korea) and cultured overnight. Media were then 
replaced with fresh conditional media (1\% FBS) containing rapamycin, and cells were allowed to grow for $48 \mathrm{~h}$. WST-1 reagent was then added to each well and cells were incubated for an additional $4 \mathrm{~h}$. Optical absorbance was measured at $450 \mathrm{~nm}$ using a microplate reader (Molecular Devices, San Jose, CA, USA), and cell counts were estimated using a standard curve.

\subsection{Immunofluorescence Microscopic Analysis}

Cells $\left(1 \times 10^{5}\right.$ cells per well) were plated in six-well culture plates (SPL Inc.,Pyeongtaek, Korea). The next day, cells were rinsed with ice-cold PBS and fixed with $4 \%$ paraformaldehyde for $20 \mathrm{~min}$ at room temperature followed by permeabilization with $0.2 \%$ Triton X-100 in PBS. The cells were subjected to immunofluorescence staining with anti-PROX1 antibody (1:300, kindly provided by Prof. Y-K Hong, University of Southern California) [25] for $1 \mathrm{~h}$ at room temperature, washed three times with cold PBS for $10 \mathrm{~min}$, and then incubated with Alexa Fluor 555-labeled anti-rabbit secondary antibody (1:800) (Santa Cruz Biotechnology, San Diego, CA, USA) at room temperature for $1 \mathrm{~h}$. Cell images were observed and captured using fluorescence microscopy (Leica, Wetzlar, Germany).

\subsection{Reverse Transcription-Polymerase Chain Reaction}

PROX1 transcript expressions were analyzed by semi-quantitative RT-PCR using human PROX1-specific primers (forward: 5'-GCAAGTTGTGGACACTGTGG-3', reverse: $5^{\prime}$ GGCAGACTGGTCAGAGGAGTT-3') and $\beta$-actin-specific-primers (forward: $5^{\prime}$-CTGGGAC GACATGGAGAAAA-3', reverse: $5^{\prime}$-AAGGAAGGCTGGAAGAGTGC-3') for quantitative comparison. Briefly, total RNA was isolated from cells using TRIzol reagent (Life Technology, Carlsbad, CA, USA) and reverse-transcribed using MMLV reverse transcriptase (Enzynomics, Daejeon, Korea) and 15 mer oligo-dT (Enzynomics, Daejeon, Korea). Prepared cDNAs were used as templates and PCR was performed according to the following protocols: (1) $94{ }^{\circ} \mathrm{C}$ for $5 \mathrm{~min}$; (2) 26 cycles at $94{ }^{\circ} \mathrm{C}$ for $30 \mathrm{~s}, 58{ }^{\circ} \mathrm{C}$ for $30 \mathrm{~s}$, and $72{ }^{\circ} \mathrm{C}$ for $1 \mathrm{~min}$; and then (3) $72{ }^{\circ} \mathrm{C}$ for $10 \mathrm{~min}$ before cooling to $4^{\circ} \mathrm{C}$. PCR products $(10 \mu \mathrm{L})$ were loaded on a $1.5 \%$ agarose gel containing ethidium bromide and visualized under UV light. Quantitative real-time PCR analysis was performed using a Step OnePlus Real-Time PCR System instrument (Applied Biosystems, Warrington, UK) using Power SYBR ${ }^{\circledR}$ Green PCR Master Mix (Applied Biosystems, Warrington, UK) using the following protocol: $50{ }^{\circ} \mathrm{C}$ for $10 \mathrm{~min}, 95^{\circ} \mathrm{C}$ for $2 \mathrm{~min}, 40$ cycles of $95^{\circ} \mathrm{C}$ for $10 \mathrm{~s}$, and final extension for $30 \mathrm{~s}$ at $55^{\circ} \mathrm{C}$.

\subsection{Protein Extraction and Western Blot Analysis}

Cells $\left(2 \times 10^{5}\right)$ were plated in $60 \mathrm{~mm}$ dishes, treated with the indicated concentrations of rapamycin for $0 \sim 48 \mathrm{~h}$, and then lysed using RIPA buffer (50 mM Tris- $\mathrm{HCl}$ [pH 7.4], $150 \mathrm{mM} \mathrm{NaCl}, 1 \%$ Nonidet P-40, 0.1\% sodium dodecyl sulfate [SDS], and 0.5\% sodium deoxycholate) containing protease and phosphatase inhibitors (Sigma-Aldrich, San Diego, CA, USA). Proteins were quantified using a Bradford Protein Assay kit (Pierce Biotechnology, Rockford, IL, USA). For Western blot assay, $20 \mu \mathrm{g}$ of proteins was separated by 10\% SDS polyacrylamide gel electrophoresis and transferred to polyvinylidene difluoride (PVDF) membranes (GE HealthCare, Hatfield, UK), which were then treated with anti-PROX1 antibody [25], anti-phospho-MTOR (p-MTOR, ser-2448) antibody (sc-101738, Santa Cruz Biotechnology, Santa Cruz, CA, USA), anti-MTOR antibody (Ab-2448, Cusabio, Houston, TX, USA), anti- $\beta$-actin antibody (sc-47778, Santa Cruz Biotechnology, CA), appropriate secondary antibodies, and enhanced chemiluminescence detection reagent (Amersham Pharmacia Biotech, Piscataway, NJ, USA).

\subsection{Analysis of PROX1 Ubiquitination Levels by Immunoprecipitation (IP)}

Cells $\left(1 \times 10^{6}\right)$ were plated in $100 \mathrm{~mm}$ dishes, treated with or without rapamycin $(10 \mathrm{nM})$ for $24 \mathrm{~h}$, and incubated with MG132 for an additional $6 \mathrm{~h}$. Total proteins were extracted using NETN buffer (100 mM NaCl, $20 \mathrm{mM}$ Tris-Cl [pH 8.0], $0.5 \mathrm{mM}$ EDTA, 0.5\% Nonidet P-40), and incubated with rabbit-IgG (1 $\mu$ g; sc-2027, Santa Cruz Biotech- 
nology) or anti-PROX1 antibody $(1 \mu \mathrm{g})$ overnight at $4{ }^{\circ} \mathrm{C}$, while appropriate amounts of extracted proteins were used as input controls. Agarose-conjugated protein $\mathrm{G}$ beads (protein G-agarose, GE-Health Care, Stockholm, Sweden) were then added and incubated for $2 \mathrm{~h}$ at $4{ }^{\circ} \mathrm{C}$. Following centrifugation for $4 \mathrm{~min}$ at $3000 \mathrm{rpm}$, the protein G-agarose beads were washed four times with $1 \mathrm{~mL}$ of lysis buffer and then boiled in protein loading buffer for $5 \mathrm{~min}$. Supernatants were subjected to Western blot analysis; ubiquitination was detected using an anti-ubiquitin antibody (sc-9133, Santa Cruz Biotechnology) and relative ubiquitination levels were determined using Image $\mathrm{J}$.

\subsection{Animal Studies}

Animal experimental protocols were preapproved by the Institutional Animal Care and Use Committee (IACUC approval number: SCH19-0032). The seven-week-old BALB/c nude mice (male) used in this study were provided by Saeronbio (Korea). Huh7 cells were suspended in serum-free DMEM media $\left(1 \times 10^{8} / \mathrm{mL}\right)$ and inoculated subcutaneously into both flanks of each mouse (100 $\mu \mathrm{L}$ each). In the first experiment, rapamycin was injected before tumors formed (40 days after inoculation). In the second, rapamycin was injected after tumor volumes reached $100 \mathrm{~mm}^{3}$. Mice were treated with vehicle (5\% PEG, $5 \%$ Tween-80 in PBS) or rapamycin ( $4 \mathrm{mg} / \mathrm{kg}$, i.p.) daily for 14 days [25-27]. Tumor volumes were calculated using the following formula: $\mathrm{V}=\mathrm{L} \times \mathrm{W}^{2} / 2$, where $\mathrm{V}=$ volume $\left(\mathrm{mm}^{3}\right)$, $\mathrm{L}=$ largest diameter $(\mathrm{mm})$, and $\mathrm{W}=$ smallest diameter $(\mathrm{mm})$. After $\mathrm{CO}_{2}$ euthanasia, tumors were extracted and fixed with $4 \%$ paraformaldehyde (PFA), following hematoxylin and eosin (H\&E) staining or immunohistochemical analyses for detecting PROX1 expression.

\subsection{Histology and Immunohistochemistry}

Fixed xenograft tissues were dehydrated in an alcohol series, treated with xylene, fused with molten paraffin infusion on an automated processor, followed by paraffinembedding, and sectioned. Immunohistochemistry was performed using a polyclonal antibody against PROX1 [25]. Sections were viewed and photographed under microscope equipped with a charge-coupled digital camera (Leica DMi8, Wetzlar, Germany). PROX1 expression was quantified using Image $\mathrm{J}$, and the results are reported as mean numbers of positive pixels/tumor area.

\subsection{Statistical Analysis}

Experimental data were analyzed using GraphPad Prism 5.0 (GraphPad Software Inc., La Jolla, CA. The results are presented as the mean \pm SDs of at least three independent experiments. One-way analysis of variance and Tukey's test were used to determine the significances of differences, and statistical significance was accepted for $p$-values $<0.05$ $\left({ }^{*} p<0.05 ;{ }^{* *} p<0.01 ;{ }^{* * *} p<0.001\right)$.

\section{Results}

\subsection{The Proliferation of Huh7 or Hep3B Cells Was Inhibited by Rapamycin}

To investigate the anti-cancer effect of rapamycin, the proliferation of HCC cell lines, Huh7, Hep3B, HepG2, or SNU3160, was measured after rapamycin treatment. Rapamycin significantly reduced the proliferation of Huh7 or Hep3B, but did not affect HepG2 or SNU3160 cells (Figure 1A), which suggests that rapamycin was not effective on all types of HCC. Rapamycin decreased the proliferation of Huh7 or Hep3B cells in a dose-dependent manner (Figure 1B). Further studies were conducted by selecting a $10 \mathrm{nM}$ concentration in which the inhibitory effect on cell proliferation was confirmed in the pilot test results using various concentrations of rapamycin. 

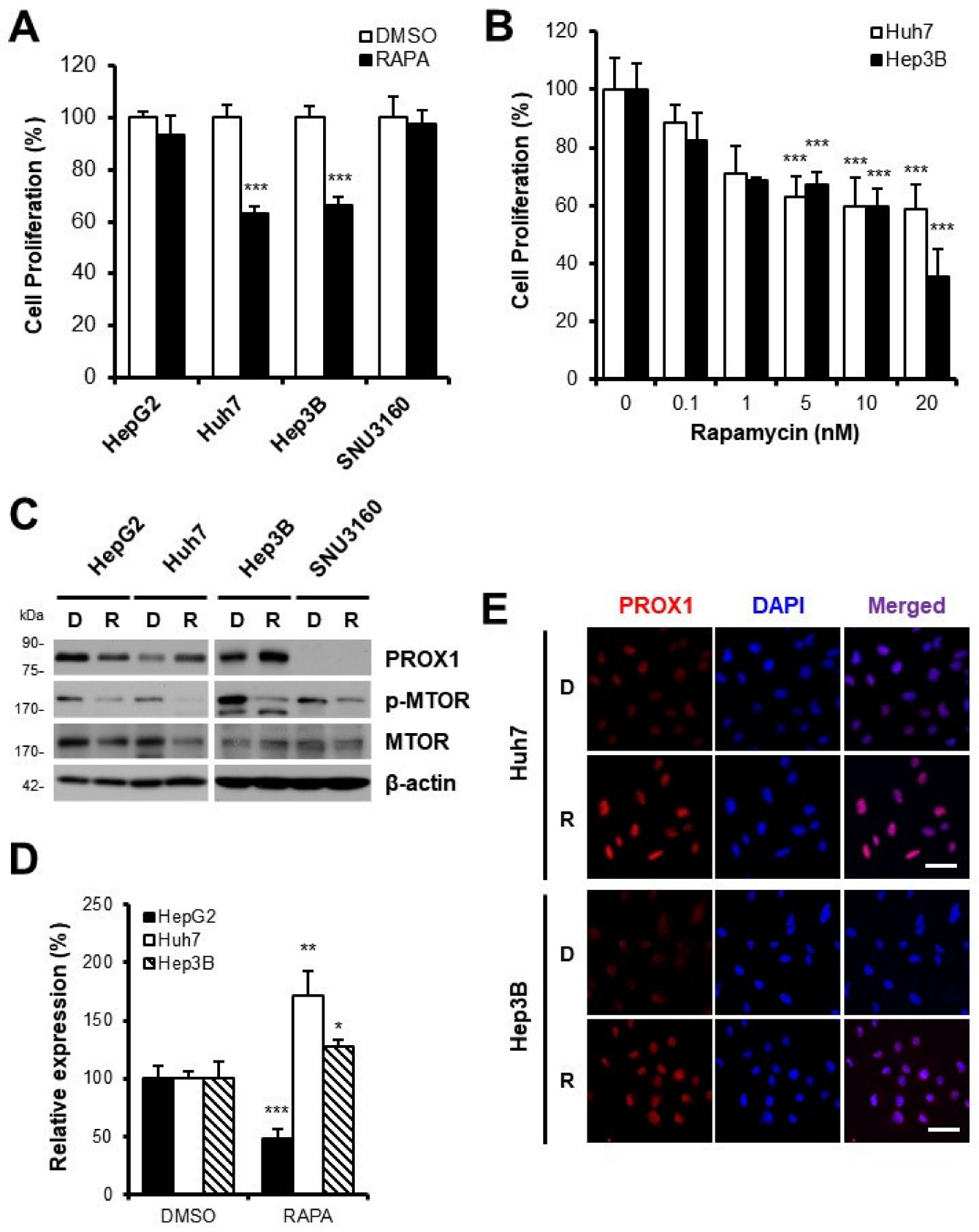

Figure 1. Effect of MTOR inhibition by rapamycin on HCC cell proliferation. Four different HCC cell lines (HepG2, Huh7, Hep3B and SNU3160) were cultured in conditional media (1\% FBS) with or without rapamycin (RAPA). Proliferation of Huh7 or Hep3B cells was inhibited by $10 \mathrm{nM}$ rapamycin for $48 \mathrm{~h}$ (A). Proliferation of Huh7 or Hep3B cells was regulated by rapamycin in a dose-dependent manner (within 0.1 20 nM) (B). PROX1 expression was increased by rapamycin in Huh7 or Hep3B cells. The inhibitory effect of rapamycin was confirmed by detecting the phosphorylated form of MTOR (p-MTOR). MTOR and $\beta$-actin were detected as quantitative controls $(\mathbf{C})$. Relative expression of PROX1 were analyzed image J (D). PROX1 expression was also assessed by immunofluorescence microscopy (E). Cell nuclei were observed by DAPI staining. D: DMSO-treated cells, R: rapamycintreated cells. Scale bars represent $100 \mu \mathrm{m} .{ }^{*} p<0.05 ;{ }^{* *} p<0.01,{ }^{* * *} p<0.001$.

Next, to investigate the effect of rapamycin on PROX1, all four HCC cells were treated with rapamycin, and PROX1 expression was assessed. The results from both Western blot and immunofluorescence analyses verified that rapamycin increased PROX1 expression in Huh7 or Hep3B, but not in HepG2 or SNU3160 cells, which was in line 
with the anti-proliferative effects of rapamycin (Figure 1C-E). On the other hand, PROX1 expression was not affected by rapamycin in HepG2 cells and not even detected in SNU3160 cells (Figure 1C,D). These results show that rapamycin increased PROX1 expression and suppressed the proliferation of Huh7 or Hep3B cells.

\subsection{PROX1 Expression in Huh7 or Hep3B Cells Was Increased by Rapamycin}

Next, the effect of rapamycin on PROX1 expression in Huh7 or Hep3B cells was measured. Although PROX1 expression was not affected by vehicle alone (DMSO), it was significantly enhanced from $12 \mathrm{~h}$ after rapamycin treatment. Phosphorylated MTOR (p-MTOR) was also detected to confirm the inhibition of MTOR by rapamycin (Huh7, Figure 2A,B; Hep3B, Figure 2E,F). PROX1 expression in cells exposed to rapamycin was increased from $1 \mathrm{nM}$ of rapamycin (Huh7, Figure 2C,D; Hep3B, Figure 2G,H). These results show that PROX1 expression was increased by rapamycin in Huh7 or Hep3B cells in a timeand dose-dependent manner.

\subsection{Increases in PROX1 Expression by Rapamycin Were Due to an Increase in Protein Half-Life}

After verifying the upregulation of PROX1 by rapamycin in Huh7 or Hep3B cells, we explored the underlying mechanisms. Initially, we examined whether this was due to transcription or protein synthesis. We found that rapamycin did not affect the expression of PROX1 mRNA in Huh7 or Hep3B cells by semi-quantitative RT-PCR (Huh7, Figure 3A; Hep3B, Figure 3E) or quantitative RT-PCR (Huh7, Figure 3B; Hep3B, Figure 3F). We then used cycloheximide ( $\mathrm{CHX})$ to examine the effect of rapamycin on levels of PROX1 protein by monitoring its half-life. Interestingly, although PROX1 levels began to decrease after $2 \mathrm{~h}$ of $\mathrm{CHX}$ treatment in control cells (DMSO), levels were maintained after CHX treatment for up to $6 \mathrm{~h}$ in rapamycin-treated Huh7 or Hep3B cells (Figure 3C,D; Figure 3G,H). These results demonstrate that enhanced PROX1 expression in rapamycin-treated Huh7 or Hep3B cells was due to increased protein stability, not increased mRNA expression.

\subsection{PROX1 Ubiquitination Was Decreased by Rapamycin}

To understand why PROX1 protein levels were increased by rapamycin, we investigated the changes in its ubiquitination. Immunoprecipitation with anti-PROX1 antibody showed the ubiquitination of PROX1 was downregulated by rapamycin in Huh7 (Figure 4A) and Hep3B cells (Figure 4C). As compared with untreated cells, PROX1 ubiquitination was decreased by $\sim 40 \%$ in Huh7 cells (Figure 4B) and by over $60 \%$ in Hep3B cells by rapamycin (Figure 4D). These data show that the higher levels of PROX1 in rapamycin-treated Huh7 or Hep3B cells were due to the suppression of PROX1 ubiquitination.

\subsection{PROX1 Played a Key Role in the Anti-Proliferative Effect of Rapamycin on Huh7 or Hep3B Cells}

To confirm the role of PROX1 in HCC proliferation, we used a PROX1 over-expression system to mimic the effect of rapamycin. Western blot analysis showed that PROX1 expression was substantially increased in both rapamycin-treated and PROX1 over-expressed cells, which confirmed the effectiveness of the over-expression system (Huh7, Figure 5A; Hep3B, Figure 5E). Notably, the proliferation of Huh7 or Hep3B cells was significantly reduced by PROX1 over-expression and by rapamycin treatment (Huh7, Figure 5B; Hep3B, Figure 5F). To investigate further the functional roles of PROX1 in HCC proliferation, we used siRNA targeting PROX1 mRNA (siPROX1). Western blot analysis confirmed that siPROX1 effectively inhibited PROX1 expression (Figure 5C,G). Next, we tested whether siPROX1 could inhibit the proliferation of cells in the presence or absence of rapamycin and found that the anti-proliferative effect of rapamycin was significantly suppressed by siPROX1 (Huh7, Figure 5D; Hep3B, Figure 5H). Collectively, these results show that PROX1 played a critical role in the anti-proliferative effect of rapamycin on Huh7 or Hep3B cells. 

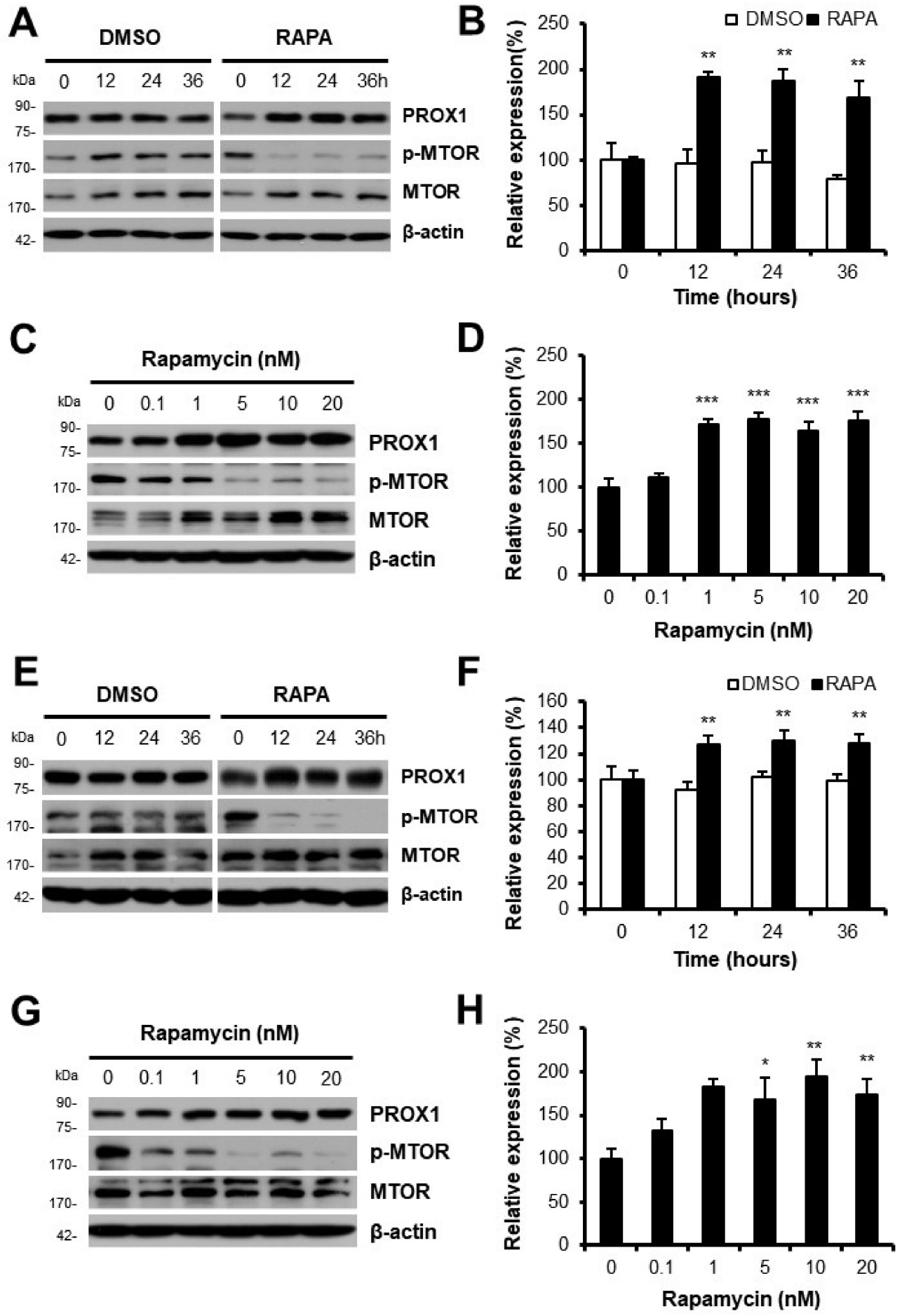

Figure 2. PROX1 expression was increased by rapamycin in Huh7 or Hep3B cells. PROX1 expression was elevated in Huh7 (A) or Hep3B cells (E) after 12 36 h of rapamycin treatment. The inhibitory effect of rapamycin was confirmed by detecting the phosphorylated form of MTOR (p-MTOR). MTOR and $\beta$-actin were detected as quantitative controls. The relative analysis comparing PROX1 expression with $\beta$-actin shows that PROX1 expression was significantly up-regulated by rapamycin in Huh7 (B) or Hep3B cells (F). Western blot analysis was performed after Huh7 (C) or Hep3B cells (G) were treated with rapamycin at concentrations of $0.1 \sim 20 \mathrm{nM}$. Rapamycin activity was confirmed by detecting p-MTOR. MTOR and $\beta$-actin were measured as quantitative controls. PROX1 expression in Huh7 (D) or Hep3B cells (H) was analyzed and compared with $\beta$-actin. ${ }^{*} p<0.05 ;{ }^{* *} p<0.01$; *** $p<0.001$. 

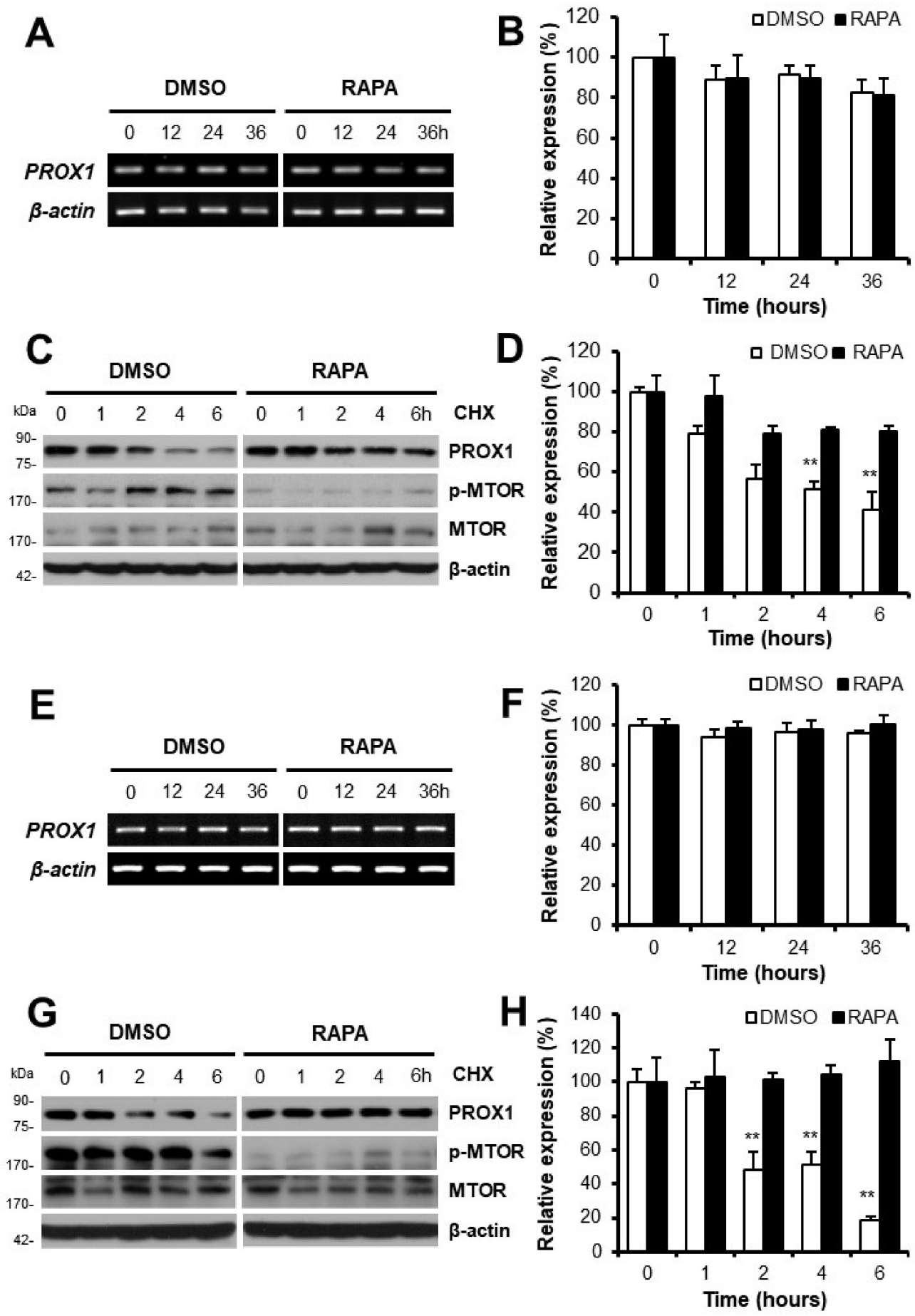

Figure 3. Rapamycin increased the intracellular half-life of PROX1 protein. The mRNA expression of PROX1 was analyzed by semi-quantitative RT-PCR using total RNAs prepared from Huh7 (A) or Hep3B cells (E) treated with or without rapamycin. Quantitative RT-PCR results showed that the expression of PROX1 mRNA in Huh7 (B) or Hep3B cells (F) was not affected by rapamycin. The half-life of PROX1 protein in Huh7 (C) or Hep3B cells (G) was measured by treating cells with or without rapamycin for $24 \mathrm{~h}$ and then exposing them to cycloheximide $(50 \mu \mathrm{g} / \mathrm{mL}$ ). Phosphorylated MTOR (p-MTOR) was detected to monitor the inhibitory effect of rapamycin. MTOR and $\beta$-actin were measured as quantitative controls. Relative analysis showed that PROX1 expression in Huh7 (D) or Hep3B cells $(\mathbf{H})$ began to decrease after $2 \mathrm{~h}$ of cycloheximide treatment in DMSO-treated control cells, but it was maintained after rapamycin treatment for up to $6 \mathrm{~h}$. ${ }^{* *} p<0.01$. DMSO: DMSO-treated cells, RAPA: rapamycin-treated cells. 
A

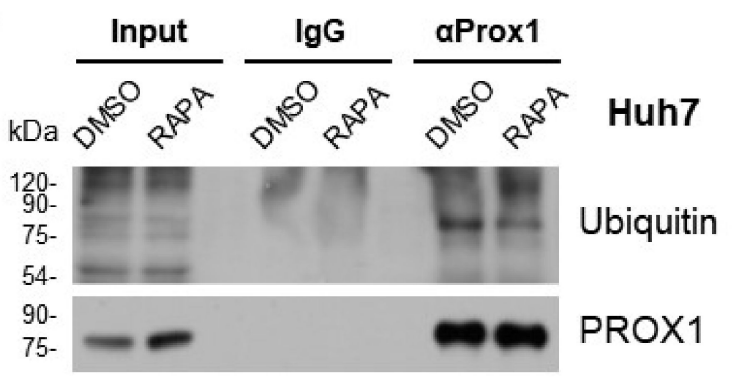

B

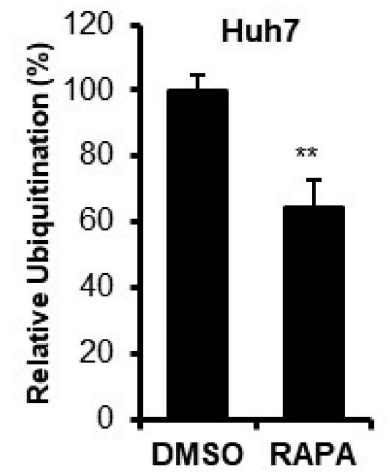

D

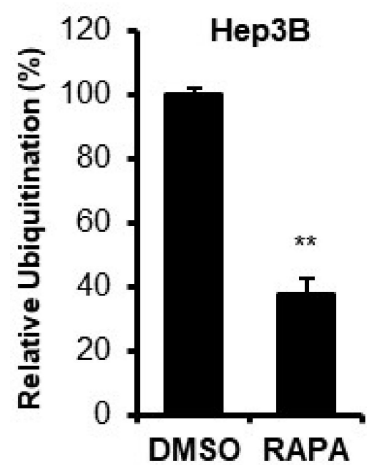

Figure 4. PROX1 ubiquitination was downregulated by rapamycin. Immunoprecipitation of PROX1 proteins was used for detection of ubiquitination. PROX1 ubiquitination was decreased after treatment with rapamycin for $24 \mathrm{~h}$ and an additional incubation with MG132 (15 $\mu \mathrm{M})$ for $6 \mathrm{~h}$ in Huh7 (A) or Hep3B (C) cells. Relative analysis showed that PROX1 ubiquitination was suppressed by rapamycin $(\mathbf{B}, \mathbf{D}) .{ }^{* *} p<0.01$. DMSO: DMSO-treated cells, RAPA: rapamycin-treated cells.

\subsection{Xenograft Tumor Growth Was Suppressed and PROX1 Expression Was Increased by} Rapamycin In Vivo

To evaluate the effect of PROX1 expression in human HCC in vivo, Huh7 cells were inoculated into the flanks of nude mice for xenograft study. In the first experiment, rapamycin was administered daily for 14 days from the 40th day when the tumor began to grow. It was found that Huh7 xenograft did not develop ( $n=10$, Figure $6 \mathrm{~A})$. In the second experiment, we injected rapamycin or vehicle daily for 14 days after the tumor volumes reached $100 \mathrm{~mm}^{3}$ and found that tumor volumes were significantly smaller in the rapamycin-treated mice than in vehicle controls (average $647 \mathrm{~mm}^{3}$ vs. $1425 \mathrm{~mm}^{3}$, $n=5$, respectively, Figure 6B,C). Subsequent immunohistochemical analyses of tumors showed that rapamycin increased PROX1 expression (Figure 6D). These in vivo results show that rapamycin suppressed Huh7 xenograft growth and increased intratumoral PROX1 expression. 
A

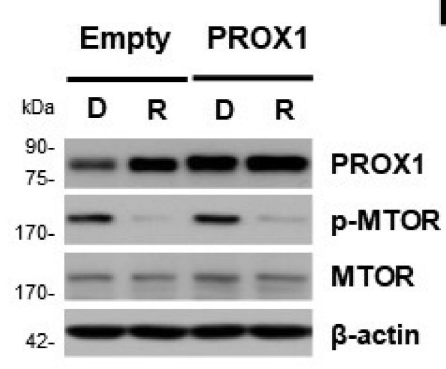

C

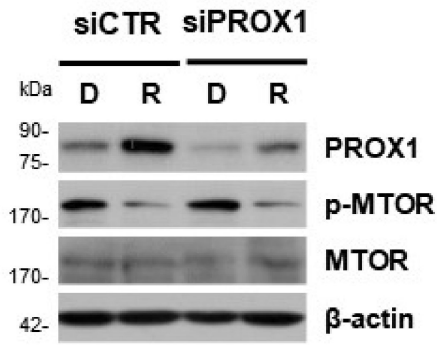

E
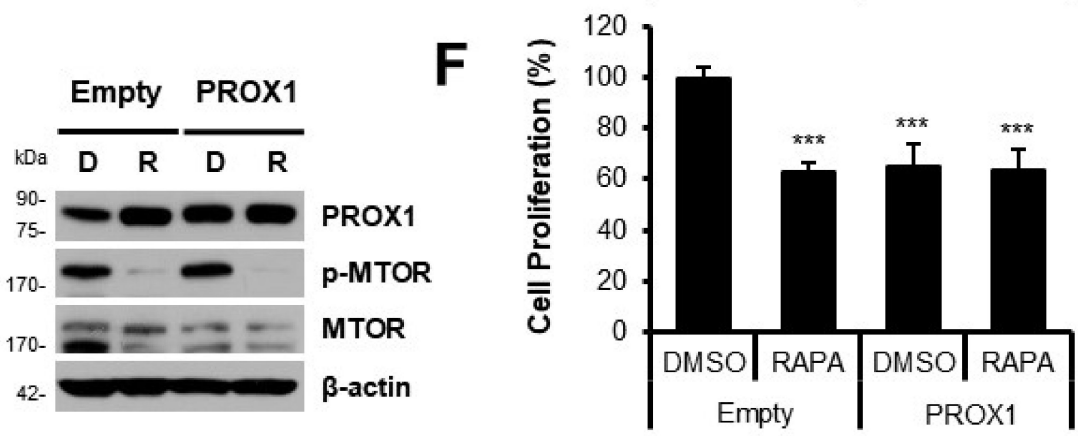

G

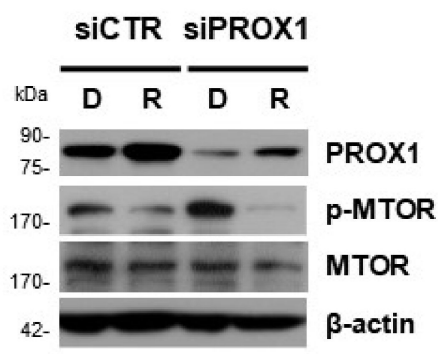

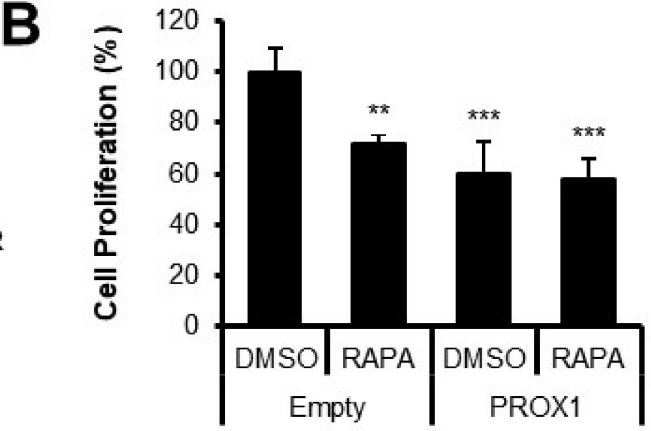

D

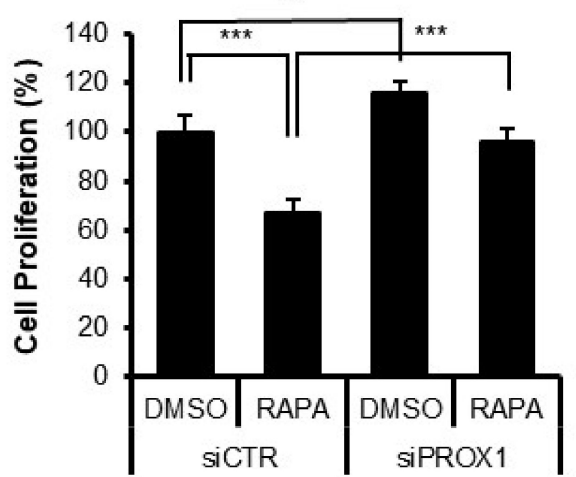

H

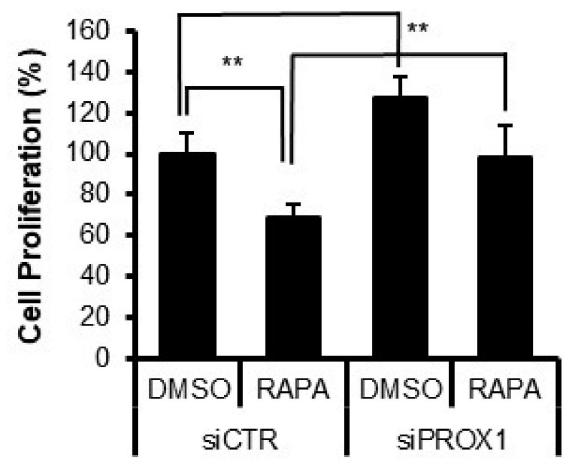

Figure 5. PROX1 played a key role in the anti-proliferative effect of rapamycin. Huh7 (A) or Hep3B cells (E) were transfected with a plasmid expressing human PROX1 or an empty plasmid, and $24 \mathrm{~h}$ later were treated with or without rapamycin for an additional $24 \mathrm{~h}$. Western blot analysis showed that PROX1 expression was increased by the over-expression system and by rapamycin, and markedly by rapamycin treatment plus PROX1 over-expression. D: cells treated with DMSO, R: cells treated with rapamycin. The effect of PROX1 on the proliferation of Huh7 (B) or Hep3B cells (F) was also investigated using the over-expression system. After $24 \mathrm{~h}$ of transfection, cells were treated with or without rapamycin for an additional $48 \mathrm{~h}$. Cell proliferation was inhibited by rapamycin and by PROX1 over-expression. Huh7 (C) or Hep3B cells (G) were transfected with siRNA targeting PROX1 mRNA (siPROX1) or control siRNA (siCTR). After $24 \mathrm{~h}$, cells were treated with or without rapamycin 
for an additional $24 \mathrm{~h}$. Western blot analysis clearly showed the knock-down effect of siPROX1. The inhibitory effect of rapamycin was confirmed by determining phosphorylated MTOR (p-MTOR). MTOR and $\beta$-actin were detected as quantitative controls. To investigate the effect of siPROX1 on cell proliferation, Huh7 (D) or Hep3B cells (H) were transfected with siPROX1 and treated with or without rapamycin for an additional $48 \mathrm{~h}$. siPROX1treatment was found to increase the proliferation of Huh7 or Hep3B cells and to decrease the anti-proliferative effect of rapamycin. ${ }^{* *}, p<0.01$; $* * *, p<0.001$. DMSO: DMSO-treated cells, RAPA: rapamycin-treated cells.

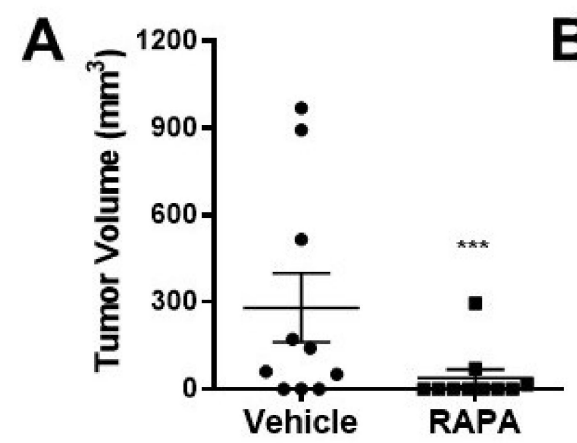

C
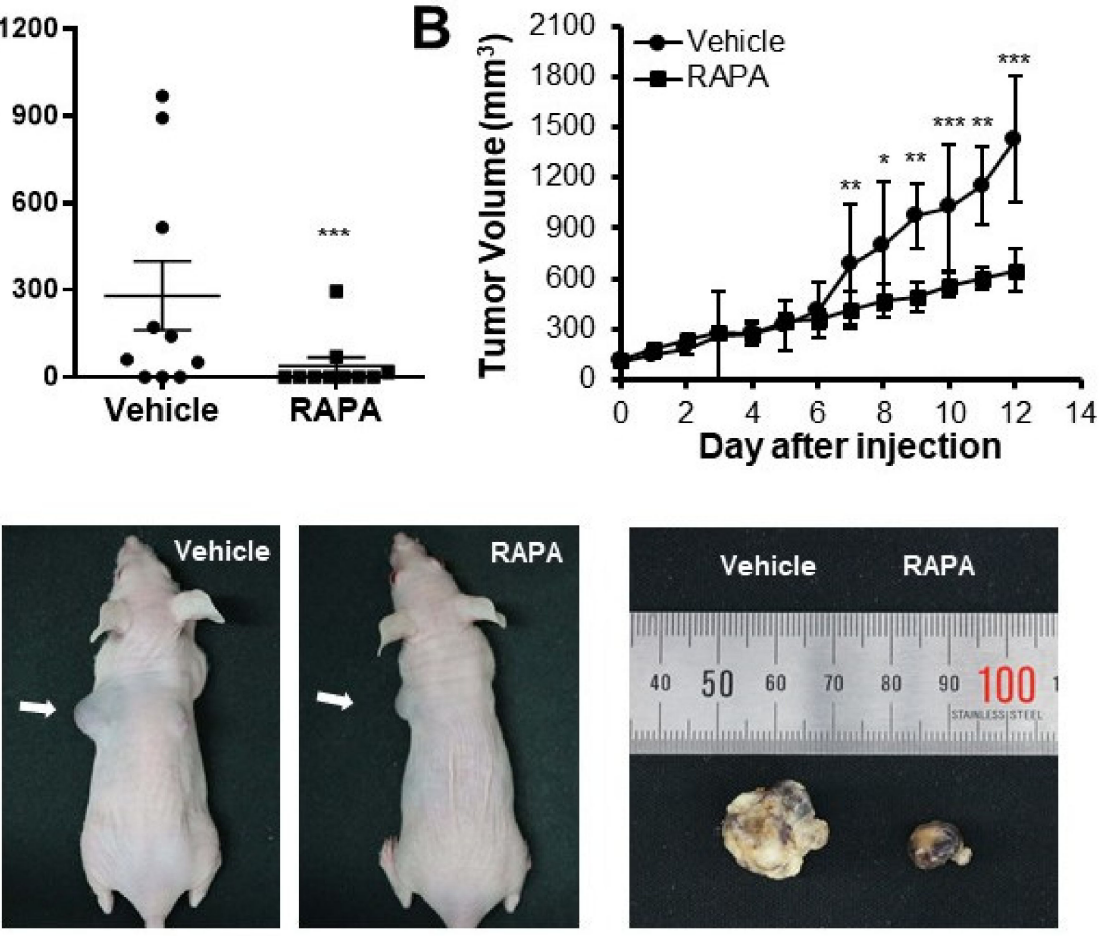

D

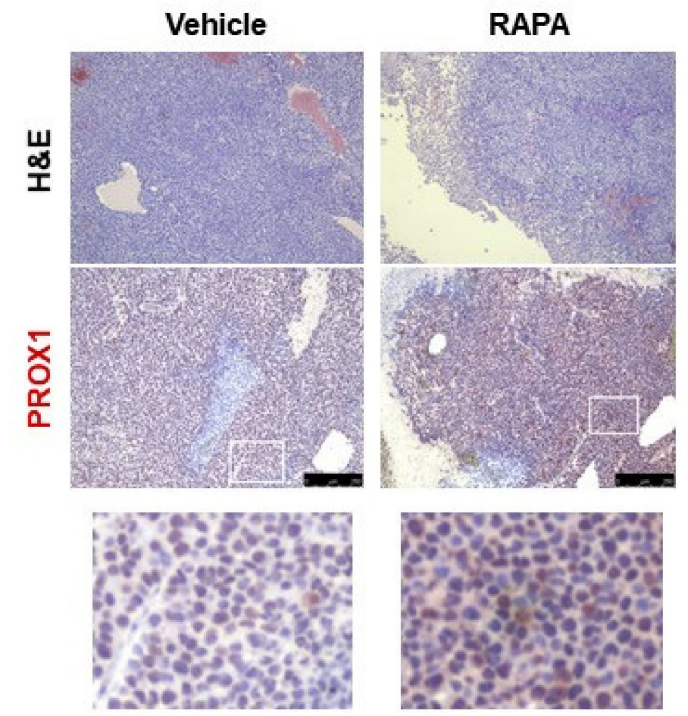

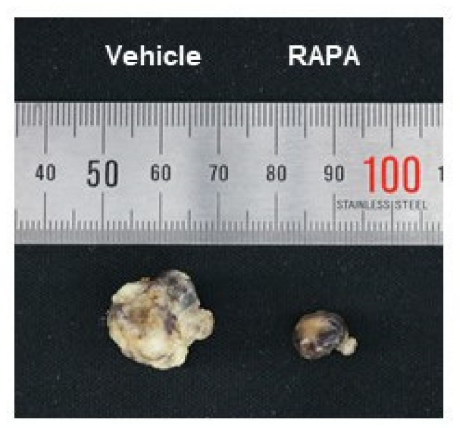

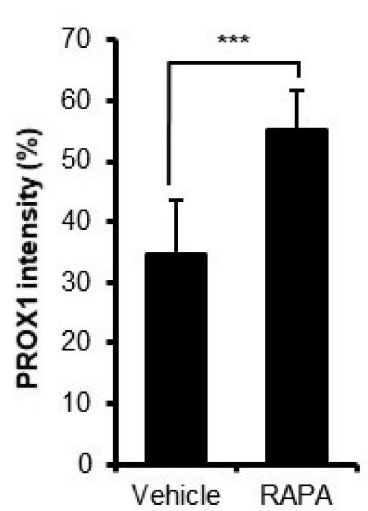

Figure 6. The effect of rapamycin on Huh7 xenograft growth and intratumoral PROX1 levels. Huh7 cells $\left(1 \times 10^{7}\right)$ were inoculated into the flanks of seven-week-old BALB/c nude mice. Tumor growth was effectively suppressed by rapamycin injected daily (at $4 \mathrm{mg} / \mathrm{kg}, \mathrm{n}=10$, respectively) for 14 days before xenograft tumor formation (A). In the second experiment, rapamycin was injected for 14 days, after the tumors reached $100 \mathrm{~mm}^{3}$ ( $\mathrm{n}=5$, respectively). Rapamycin suppressed tumor growth $(\mathbf{B}, \mathbf{C})$ and increased PROX1 levels (D). ${ }^{*} p<0.05 ;{ }^{* *} p<0.01 ;{ }^{* * *} p<0.001$. 


\section{Discussion}

HCC is characterized by several genetic changes that affect multiple signal pathways and result in the uncontrolled proliferation of hepatocytes [28]. Rapamycin inhibits MTOR and has been widely used to prevent the rejection of transplanted organs [16]. In addition to being used as an immunosuppressant, the clinical applications of rapamycin have been extended to cancer treatment. Recently, MTOR-specific inhibitors were examined as potential anti-cancer agents and showed anti-cancer activity in several patients with advanced HCC [29]. However, a clinical trial (EVOLVE-1) of the MTOR inhibitor, everolimus, showed that it did not improve overall survival in advanced HCC patients unresponsive or intolerant to sorafenib [30]. Therefore, a suitable chemotherapy agent that specifically targets a molecule required by HCC is urgently required to enable the development of personalized targeted therapy.

Here, we investigated the anti-proliferative effect of rapamycin, an MTOR inhibitor, on four different HCC cell lines, Huh7, Hep3B, HepG2, and SNU3160. SNU3160 cells are WHO grade G2 (moderately differentiated), HBV negative, and PROX1-negative. Interestingly, the anti-proliferative effects of rapamycin were only apparent in Huh7 or Hep3B (Figure 1A), which was probably owing to their heterogeneous origins. Indeed, it is well known that HCC has a heterogeneous etiology, and thus only some HCC patients benefit from sorafenib treatment. The anti-proliferative effect of MTOR inhibition on Huh7 or Hep3B cells was confirmed with temsirolimus, another MTOR inhibitor (data not shown).

During our investigation to determine whether underlying molecular mechanisms might explain these results, it was observed that the anti-proliferative effect appeared only in cell lines where PROX1 protein increased after rapamycin treatment Although rapamycin treatment significantly and time- and dose-dependently increased PROX1 expression in Huh7 or Hep3B cells (Figure 2), interestingly, rapamycin suppressed PROX1 expression in HepG2 cells (Figure 1C,D). We previously reported the inhibitory effect of rapamycin on PROX1 expression, and resultant triglyceride accumulation in hepatocytes [25]. Notably, PROX1 protein was not detected in SNU3160 cells (Figure 1C). In addition, cell proliferation was not affected by high concentrations of rapamycin, but was inhibited by PROX1 overexpression in these two cell lines (data not shown).

As there are few reports showing the relationship between the MTOR signal and PROX1, further investigation is needed to identify the mechanisms by which the MTOR signal affects PROX1 expression and to determine the causes of different effects of rapamycin on PROX1 expression among HCC cell lines. To understand the upregulation of PROX1 by MTOR inhibition, changes in its mRNA expression were first investigated. Although the mRNA expression of PROX1 was not affected, the half-life of PROX1 protein was enhanced by rapamycin in Huh7 or Hep3B cells (Figure 3), which was achieved by reduced PROX1 ubiquitination (Figure 4). The upregulation of PROX1 protein might be related to the anti-proliferative effect of rapamycin in Huh7 or Hep3B cells. Because there are few studies reporting the process of PROX1 ubiquitination, further studies are required to identify and investigate factors related to PROX1PROX1 ubiquitination, and further studies are required to identify and investigate factors related to PROX1 ubiquitination, such as E3 ligase.

To verify the participation of PROX1 in the anti-proliferative effect of rapamycin, PROX1 over-expression was used to mimic the effect of rapamycin in Huh7 or Hep3B cells. Cell proliferation was downregulated by PROX1 over-expression in both cell lines in a manner similar to that of rapamycin (Huh7, Figure 5B; Hep3B, Figure 5F). Interestingly, the loss-of-function experiment using PROX1 siRNA showed that the anti-proliferative effect of rapamycin was abrogated by PROX1 knock-down (Figure 5D,H). PROX1 protein levels were not affected by rapamycin in Huh7 and Hep3B cells transfected with the PROX1expressing vector, which means that the effect of MTOR inhibition on PROX1 expression was diminished by the overexpression system.

To check the anti-cancer effect of PROX1 and MTOR inhibition in vivo, Huh7 cells were inoculated into the flanks of nude mice. In the first experiment, rapamycin was injected into mice before xenografts formed, and as expected, tumor development was suppressed 
well by rapamycin (Figure 6A). In the second experiment, rapamycin was injected when the tumors volume exceeded $100 \mathrm{~mm}^{3}$. Again, tumor growth was suppressed by rapamycin (Figure 6B,C), and immunohistochemical analyses showed intratumoral PROX1 levels were increased by rapamycin (Figure 6D). These results indicate that increased PROX1 expression was related to the anti-proliferative effect of rapamycin on HCC.

In conclusion, we found that rapamycin, an MTOR inhibitor, reduced the proliferation of Huh7 or Hep3B cells by increasing the half-life of PROX1 protein. Functional studies using an over-expression, or a knock-down, system showed that PROX1 critically mediates the anti-proliferative effect of rapamycin on Huh7 or Hep3B cells. Furthermore, our xenograft study revealed that the growth of Huh7 xenografts was suppressed and that PROX1 expression was increased by rapamycin. Together, these results suggest that PROX1 plays a critical role in the anti-proliferative effect of rapamycin on HCC and that the upregulation of PROX1 by an MTOR inhibitor might be a useful molecular marker for predicting the efficacy of its anti-cancer effect on HCC cells.

Author Contributions: Conceptualization, S.K. and J.-S.S.; Data curation, S.K.; Funding acquisition, I.C.; Investigation, S.K.; Supervision, I.C.; Writing—original draft, S.K. and I.C.; Writing-review \& editing, K.B. and Y.-K.H. All authors have read and agreed to the published version of the manuscript.

Funding: This research was funded by the Ministry of Education, National Research Foundation of Korea (NRF, grant number [2017R1D1A1B03028573]).

Institutional Review Board Statement: Animal experimental protocols were preapproved by the Institutional Animal Care and Use Committee (IACUC approval number: SCH19-0032).

Informed Consent Statement: Not applicable.

Data Availability Statement: Not applicable.

Acknowledgments: This research was supported by Basic Science Research Program through the National Research Foundation of Korea (NRF) funded by the Ministry of Education (2017R1D1A1B03028573).

Conflicts of Interest: The authors declare no conflict of interest.

\section{References}

1. Svinka, J.; Mikulits, W.; Eferl, R. STAT3 in Hepatocellular Carcinoma: New Perspectives. Hepatic Oncol. 2014, 1, 107-120. [CrossRef] [PubMed]

2. Sung, H.; Ferlay, J.; Siegel, R.L.; Laversanne, M.; Soerjomataram, I.; Jemal, A.; Bray, F. Global Cancer Statistics 2020: GLOBOCAN Estimates of Incidence and Mortality Worldwide for 36 Cancers in 185 Countries. CA Cancer J. Clin. 2021, 71, 209-249. [CrossRef] [PubMed]

3. Huang, A.; Yang, X.-R.; Chung, W.-Y.; Dennison, A.R.; Zhou, J. Targeted Therapy for Hepatocellular Carcinoma. Signal Transduct. Target. Ther. 2020, 5, 146. [CrossRef] [PubMed]

4. $\quad$ Cheng, A.-L.; Guan, Z.; Chen, Z.; Tsao, C.-J.; Qin, S.; Kim, J.S.; Yang, T.-S.; Tak, W.Y.; Pan, H.; Yu, S.; et al. Efficacy and Safety of Sorafenib in Patients with Advanced Hepatocellular Carcinoma According to Baseline Status: Subset Analyses of the Phase III Sorafenib Asia-Pacific Trial. Eur. J. Cancer 2012, 48, 1452-1465. [CrossRef]

5. Kudo, M.; Finn, R.S.; Qin, S.; Han, K.-H.; Ikeda, K.; Piscaglia, F.; Baron, A.; Park, J.-W.; Han, G.; Jassem, J.; et al. Lenvatinib versus Sorafenib in First-Line Treatment of Patients with Unresectable Hepatocellular Carcinoma: A Randomised Phase 3 Non-Inferiority Trial. Lancet 2018, 391, 1163-1173. [CrossRef]

6. Hiraoka, A.; Kumada, T.; Tada, T.; Tani, J.; Kariyama, K.; Fukunishi, S.; Atsukawa, M.; Hirooka, M.; Tsuji, K.; Ishikawa, T.; et al. Efficacy of Lenvatinib for Unresectable Hepatocellular Carcinoma Based on Background Liver Disease Etiology: Multi-Center Retrospective Study. Sci. Rep. 2021, 11, 16663. [CrossRef]

7. Laplante, M.; Sabatini, D.M. MTOR Signaling at a Glance. J. Cell Sci. 2009, 122, 3589-3594. [CrossRef]

8. Mita, M.M.; Mita, A.; Rowinsky, E.K. Mammalian Target of Rapamycin: A New Molecular Target for Breast Cancer. Clin. Breast Cancer 2003, 4, 126-137. [CrossRef]

9. Xu, G.; Zhang, W.; Bertram, P.; Zheng, X.F.; McLeod, H. Pharmacogenomic Profiling of the PI3K/PTEN-AKT-MTOR Pathway in Common Human Tumors. Int. J. Oncol. 2004, 24, 893-900. [CrossRef]

10. Sahin, F.; Kannangai, R.; Adegbola, O.; Wang, J.; Su, G.; Torbenson, M. MTOR and P70 S6 Kinase Expression in Primary Liver Neoplasms. Clin. Cancer Res. 2004, 10, 8421-8425. [CrossRef]

11. Xu, X.; Sakon, M.; Nagano, H.; Hiraoka, N.; Yamamoto, H.; Hayashi, N.; Dono, K.; Nakamori, S.; Umeshita, K.; Ito, Y.; et al. Akt2 Expression Correlates with Prognosis of Human Hepatocellular Carcinoma. Oncol. Rep. 2004, 11, 25-32. [CrossRef] 
12. Davies, S.P.; Reddy, H.; Caivano, M.; Cohen, P. Specificity and Mechanism of Action of Some Commonly Used Protein Kinase Inhibitors. Biochem. J. 2000, 351, 95-105. [CrossRef]

13. Dutcher, J.P. Mammalian Target of Rapamycin (MTOR) Inhibitors. Curr. Oncol. Rep. 2004, 6, 111-115. [CrossRef]

14. Kahan, B.D. The Limitations of Calcineurin and MTOR Inhibitors: New Directions for Immunosuppressive Strategies. Transplant. Proc. 2002, 34, 130-133. [CrossRef]

15. Hidalgo, M.; Rowinsky, E.K. The Rapamycin-Sensitive Signal Transduction Pathway as a Target for Cancer Therapy. Oncogene 2000, 19, 6680-6686. [CrossRef]

16. De Fijter, J.W. Cancer and MTOR Inhibitors in Transplant Recipients. Transplantation 2017, 101, 45-55. [CrossRef]

17. Wigle, J.T.; Oliver, G. Prox1 Function Is Required for the Development of the Murine Lymphatic System. Cell 1999, 98, 769-778. [CrossRef]

18. Lee, S.; Choi, I.; Hong, Y.-K. Heterogeneity and plasticity of lymphatic endothelial cells. Semin. Thromb. Hemost. 2010, 36, 352-361. [CrossRef]

19. Choi, I.; Lee, S.; Hong, Y.-K. The New Era of the Lymphatic System: No Longer Secondary to the Blood Vascular System. Cold Spring Harb. Perspect. Med. 2012, 2, a006445. [CrossRef]

20. Elsir, T.; Smits, A.; Lindström, M.S.; Nistér, M. Transcription Factor PROX1: Its Role in Development and Cancer. Cancer Metastasis Rev. 2012, 31, 793-805. [CrossRef]

21. Liu, Y.; Ye, X.; Zhang, J.-B.; Ouyang, H.; Shen, Z.; Wu, Y.; Wang, W.; Wu, J.; Tao, S.; Yang, X.; et al. PROX1 Promotes Hepatocellular Carcinoma Proliferation and Sorafenib Resistance by Enhancing $\beta$-Catenin Expression and Nuclear Translocation. Oncogene 2015, 34, 5524-5535. [CrossRef]

22. Chang, T.-M.; Hung, W.-C. The Homeobox Transcription Factor Prox1 Inhibits Proliferation of Hepatocellular Carcinoma Cells by Inducing P53-Dependent Senescence-like Phenotype. Cancer Biol. Ther. 2013, 14, 222-229. [CrossRef]

23. Rodrigues, M.F.S.D.; de Oliveira Rodini, C.; de Aquino Xavier, F.C.; Paiva, K.B.; Severino, P.; Moyses, R.A.; López, R.M.; DeCicco, R.; Rocha, L.A.; Carvalho, M.B.; et al. PROX1 Gene Is Differentially Expressed in Oral Cancer and Reduces Cellular Proliferation. Medicine 2014, 93, e192. [CrossRef]

24. Kwon, S.; Jeon, J.-S.; Ahn, C.; Sung, J.-S.; Choi, I. Rapamycin Regulates the Proliferation of Huh7, a Hepatocellular Carcinoma Cell Line, by up-Regulating P53 Expression. Biochem. Biophys. Res. Commun. 2016, 479, 74-79. [CrossRef]

25. Kwon, S.; Jeon, J.-S.; Kim, S.B.; Hong, Y.-K.; Ahn, C.; Sung, J.-S.; Choi, I. Rapamycin Up-Regulates Triglycerides in Hepatocytes by down-Regulating Prox1. Lipids Health Dis. 2016, 15, 41. [CrossRef]

26. Phung, T.L.; Ziv, K.; Dabydeen, D.; Eyiah-Mensah, G.; Riveros, M.; Perruzzi, C.; Sun, J.; Monahan-Earley, R.A.; Shiojima, I.; Nagy, J.A.; et al. Pathological Angiogenesis Is Induced by Sustained Akt Signaling and Inhibited by Rapamycin. Cancer Cell 2006, 10, 159-170. [CrossRef]

27. Banerjee, S.; Gianino, S.M.; Gao, F.; Christians, U.; Gutmann, D.H. Interpreting Mammalian Target of Rapamycin and Cell Growth Inhibition in a Genetically-Engineered Mouse Model of Nf1-Deficient Astrocytes. Mol. Cancer Ther. 2011, 10, 279-291. [CrossRef]

28. Maurya, A.K.; Vinayak, M. Anticarcinogenic Action of Quercetin by Downregulation of Phosphatidylinositol 3-Kinase (PI3K) and Protein Kinase C (PKC) via Induction of P53 in Hepatocellular Carcinoma (HepG2) Cell Line. Mol. Biol. Rep. 2015, 42, 1419-1429. [CrossRef]

29. Decaens, T.; Luciani, A.; Itti, E.; Hulin, A.; Roudot-Thoraval, F.; Laurent, A.; Zafrani, E.S.; Mallat, A.; Duvoux, C. Phase II Study of Sirolimus in Treatment-Naive Patients with Advanced Hepatocellular Carcinoma. Dig. Liver Dis. 2012, 44, 610-616. [CrossRef]

30. Zhu, A.X.; Kudo, M.; Assenat, E.; Cattan, S.; Kang, Y.-K.; Lim, H.Y.; Poon, R.T.P.; Blanc, J.-F.; Vogel, A.; Chen, C.-L.; et al. Effect of Everolimus on Survival in Advanced Hepatocellular Carcinoma after Failure of Sorafenib: The EVOLVE-1 Randomized Clinical Trial. JAMA 2014, 312, 57-67. [CrossRef] 